Instituto Internacional de Investigación y Desarrollo Tecnológico Educativo INDTEC, C.A.

DOI: https://doi.org/10.29394/Scientific.issn.2542-2987.2019.4.11.18.341-359

OAI-PMH: http://www.indteca.com/ojs/index.php/Revista Scientific/oai

Ensayo Original / Original Essay

\title{
La Argumentación como Modo de Organización Discursiva en la Construcción del Genero Ensayo
}

Autora: Magdaly Carolina Méndez Omaña

Universidad Fermín Toro, UFT

mgdy.m41@gmail.com

Mérida, Venezuela

http://orcid.org/0000-0003-2931-4479

\section{Resumen}

En el presente ensayo se expone la relevante temática de la argumentación en la construcción del género ensayo en la que se involucra una comunidad efectiva, permitiendo la comunicación entre las personas con una lengua en común que trascienda las particularidades históricas y locales, conllevando a que las ideas expuestas en la producción argumentativa deben inducir a pensamientos generalizadores de temas circundantes, que se lleva a cabo en el discurso intersubjetivo. En tal sentido, el objetivo general de la investigación es generar a partir del análisis del discurso y de la didáctica de la lengua una propuesta pedagógica para la construcción del género ensayo en el nivel de educación media general. Para ello, el estudio se inserta en el paradigma interpretativo con un enfoque metodológico cualitativo. En correspondencia con la temática se puede afirmar que la argumentación es de uso común y espontáneo en la vida diaria y en las prácticas educativas, incluso sin percatarse de ello. Sin embargo, cuando se hace consciente el propósito de comunicar las ideas en forma argumentada es para su posible comprensión, esta argumentación estaría estructurada en las intenciones de los sujetos discursivos y en los intereses de los interpretantes, ya que sería válido cierto argumento o no de acuerdo con las características de los propios interlocutores con relación a sus creencias históricas y culturales. Se plantea entonces que en las prácticas educativas en especial en el nivel de media general se intenta construir el género ensayo con el propósito de comunicar. No obstante, no se hace énfasis en la argumentación como modo de organización discursiva.

Palabras clave: escritura; lingüística; pedagogía; ensayo. 


\title{
The Argumentation as a Discursive Organization Mode in the Construction of the Essay Genre
}

\begin{abstract}
This essay exposes the thematic relative of the argumentation in the construction of the essay genre in which an affective community is involved, allowing the communication between people with a common language that transfers the historical and local hardware, conducting that the ideas exposed in the argumentative production should induce the generalizing thoughts of circumbient themes, which is carried out in the intersubjective speech. in such sense, the general objective of the investigation is to generate from the analysis of the discourse and the didactics of the language, a pedagogical proposal for the construction of the genre essay at the general media education level. For this, the study is inserted in the interpretative paradigm with a qualitative methodological approach. in correspondence with the theme it is possible to affirm that the argumentation is of common and spontaneous use in the daily variation and in the educational practices, included without percating of it. however, when the purpose of communicating the ideas is aware in an argumented form is for their possible understanding, this argumentation would be structured in the intentions of the discussing subjects and in the interests of the interpreters, since some argument would be valid or not, according to the characteristics of the own partners in relation to their historical and cultural beliefs. it is therefore raised that in educational practices, especially at the general media level, an intended building of a gender test for the purpose of communicating. However, no emphasis is made in the argumentation as a mode of discursive organization.
\end{abstract}

Keywords: writing; linguistics; pedagogy; testing. 


\section{Introducción}

Este ensayo es parte de la investigación doctoral en proceso que tiene como propósito: generar a partir del análisis del discurso y de la didáctica de la lengua una propuesta pedagógica para la construcción del género ensayo en el nivel de educación media general. Se basa en una revisión teórica acerca de la argumentación como modo de organización discursiva en el género ensayo, así como la manera para propiciar la práctica argumentativa en la producción escrita. Con relación a ello, se fundamentará en los elementos teóricos, pedagógicos y didácticos de la lengua.

Atendiendo a lo anterior, se plantean propósitos relacionados al problema central de la investigación, el cual se vislumbra como la ausencia de la práctica argumentativa como modo de organización discursiva en la producción escrita, en este caso en la construcción del género ensayo, esta dificultad se presenta en los docentes de educación media general, los cuales no hacen uso de las bondades de la argumentación en la elaboración de textos escritos por parte de los estudiantes.

Es evidente que se hace necesaria la búsqueda de una alternativa que coadyuve a la situación descrita, para ello se señala la propuesta pedagógica basada en la argumentación como modo de organización discursiva para la construcción del género ensayo, hecho este que contribuye en afianzar la formación docente y en permitir a los estudiantes mejorar su proceso de aprendizaje.

Asimismo, en la construcción del género ensayo se puede considerar el modo de organización discursiva inherente en la argumentación. Al respecto Charaudeau (2004a), señala: "se trata aquí de un conjunto de procedimientos lo que yo mismo he llamado modos de organización del discurso (narrativo, descriptivo, argumentativo), que deben ser considerados como mecanismo de construcción del discurso" (pág. 26).

Estas consideraciones, presentan a la argumentación como un modo 
de organización que en la construcción de ensayos se configura en una herramienta necesaria para acceder a los saberes organizados que forman parte de la cultura y por ello se señala la importancia de la enseñanza de la argumentación y de sus técnicas.

\section{Desarrollo}

\subsection{La Argumentación Retórica}

Se entiende por la argumentación retórica el proceso discursivo por el cual un orador logra atraer la atención del auditorio con el propósito de convencer o persuadir sobre determinado tema que se argumenta con premisas probables con relación a un sistema de valores para llegar a unas conclusiones.

Martínez (2005a): expone que "en el año 1958 el teórico Perelman, renueva la retórica y la argumentación en Europa, después de que estas disciplinas estuvieron destinadas solo al tratamiento estilístico o género epidíctico literario, basados en las pruebas lógicas de la verdad" (pág. 160). Para ello, Perelman, propone que en el campo de la argumentación no es el de la certeza del cálculo, sino de lo verosímil, plausible, o probable, escenario en el que el orador pretende conseguir la adhesión, la intensidad variable del auditorio. Existen, dice Perelman (1997a): "otros tipos de razonamientos diferentes de los propuestos por la lógica clásica y que, sin embargo, no son ilógicos" (pág. 162). Esto, conduce a controversias de los métodos lógicos experimentales, los que no logran establecer acuerdos, éstos se sitúan en el campo de la deliberación, de la discusión, es decir, en el marco de la argumentación.

\subsection{La Retórica Aristotélica}

La retórica de Aristóteles incluye la dialéctica, la cual se presenta como un diálogo de lo probable, en la que se establece la definición tripartita del 
Ethos, el Logos y pathos. Andarcia (2006), aporta lo siguiente: "de los argumentos procurados por el discurso hay tres especies. Unos residen en el comportamiento del habla (ethos): otros, en poner al oyente en una determinada disposición (pathos) otros, en el propio discurso (logos)" (pág. 32). A partir de ello, se diferenció tres tipos de discursos: el deliberativo, el judicial y el epidíctico.

Con relación al género judicial, la retórica antigua lo consideraba como un evento comunicativo basado en acusaciones sobre lo justo o lo injusto, evaluadas ante un juez y el auditorio propuesto era un tribunal. En cuanto al género epidíctico hace referencia inicialmente a las honras fúnebres, y en la actualidad se pudiera ampliar el referente a actos conmemorativos culturales, políticos y representativos de la sociedad.

En este orden de ideas también se halla el género deliberativo el cual se construye en los discursos de las asambleas públicas en búsqueda de lo útil para la comunidad. En tal sentido, lo expuesto establece una relación importante entre los elementos que conforman un evento comunicativo argumentativo en la construcción del discurso. Debe señalarse que para este filósofo, la retórica cubre tres campos: una la teoría de la argumentación, que constituye su eje central y que proporciona al mismo tiempo el nudo de su articulación con la lógica demostrativa de la filosofía, una teoría de la elocuencia y una teoría de los tropos.

En este supuesto, Aristóteles intenta referenciar que la retórica concebida como el arte de persuadir y convencer dejan de ser solo ornamentos en los textos escritos ya sean poéticos o no, y se conforman con el discurso, es decir, la retórica como técnica de la comunicación y del discurso persuasivo. Para agregar a lo ya descrito el razonamiento sobre el tema de la argumentación es dialéctico si las premisas están constituidas por opiniones generalmente aceptadas.

Perelman (1997b), las define así: "las opiniones generalmente 
aceptadas por todos, por la mayor parte o por los más notables..." (pág. 23); de ello se infiere que un argumento persuasivo es el que persuade aquel a quien se dirige el mismo, en el texto referido se esboza los pensamientos que expone Aristóteles sobre razonamientos lógicos y razonamientos dialécticos haciendo una diferencia entre verdad y opinión. Esto, hace énfasis en la adhesión entre el orador y el auditorio a quien se dirige el argumento, hecho éste imprescindible en el logro de la efectividad de producción argumentativa.

\subsection{La Argumentación}

Para efectos de este trabajo se define como la habilidad de expresarse de forma oral o escrita con un propósito definido tomando en cuenta el contexto social y cultural del evento comunicativo, el interés de los interlocutores para lograr convencer o persuadir sobre alguna situación discursiva y así causar un impacto positivo en el otro. Para Calsamiglia y Tusón (2001):

La argumentación es una práctica discursiva que responde que responde a una función comunicativa: la que se orienta al receptor para lograr una adhesión. Muchos son los discursos que incluyen esta función: el discurso de las personas que se dedican a la enseñanza, a la política, a la predicación, a escribir ensayos que quieren influir (pág. 294).

Por su parte, Charaudeau (2004b), expone la argumentación como modo de organización discursiva, el cual es para este articulo el eje central y constructo temático del mismo. Al respecto, el autor antes citado aporta lo siguiente:

Las categorías prototípicas homogéneas que constituyen esta estructura son "secuencias autónomas" (relato, descripción, explicación, argumentación y diálogo) cuya configuración se descubre por los haces de las regularidades que se encuentran en el texto. Para otros, se trata aquí de un conjunto de procedimientos, lo que yo mismo he llamado "modos de organización del discurso", que deben ser considerados como mecanismos de construcción del discurso de los cuales 
dispondrá el sujeto hablante para organizar su intención discursiva, y no como el esquema del texto (pág. 26).

Sobre la base de las ideas expuestas se puede considerar la argumentación como un elemento discursivo de gran importancia para expresarnos ya sea de forma escrita o de forma oral y que permite al sujeto hablante exponer sus ideas, opiniones, percepciones, entre otros, con una ilación coherente y pertinente a un evento comunicativo determinado por un propósito social determinado por los interlocutores.

\subsection{Definición de género discursivo}

Al igual que la sociedad, el lenguaje también cambia y con estos cambios los géneros discursivos, se han diversificado, definidos a partir de la situación social de habla dominante. En función de ello, se identifican las diferencias entre un escrito que se ubica en el género pedagógico, de divulgación científica y de género científico o publicitario, religiosos, entre otros, o sencillamente se diferencia entre una conversación familiar de una entrevista o reportaje televisivo.

Además, es posible diferenciar dentro de un mismo género discursivo las formas diversas de la enunciación que son los responsables de las construcciones textuales; para ejemplificar lo anterior en el género epistolar, hay diferentes formas de enunciación como: carta familiar, y carta formal administrativa, otro ejemplo, en el género periodístico se encuentran las crónicas, reportajes, e informaciones, entre otros.

De igual manera, los géneros privilegian modos de organización diferentes: en el género literario en específico la novela, la organización por excelencia es la narrativa, aunque también se encuentra la argumentativa, expositiva, explicativa y dialogal. Otro ejemplo que se puede considerar es el género pedagógico en el que se privilegia la explicación que según al público 
que se dirige varía la organización narrativa si es educación primaria y explicativa o expositiva en constantes construcciones a través de ensayos en el nivel de media general y universitario.

Es oportuno considerar que, cuando se trata de narración, argumentación y explicación se trata de formas o modos de organización y no de géneros discursivos. El aporte que realiza Martínez (2005b), al respecto:

Los géneros discursivos se relacionan con las prácticas sociales humanas: habrá tantos géneros discursivos que no existen en unas sociedades y si en otras, pues en aquellas no hay prácticas sociales que existan en estas. Por ejemplo, hay comunidades donde no existe el género científico o, el género pedagógico institucional, allí los géneros más utilizados son el conversacional y el relato cotidiano (pág. 59).

Según lo descrito hay una evidente relación entre prácticas sociales y uso del lenguaje, en tanto que los géneros discursivos, forman parte fundamental de las construcciones discursivas de las sociedades y los sujetos discursivos de estas mismas sociedades.

\subsection{El ensayo y la construcción de la identidad discursiva}

Todo texto ya sea oral o escrito se inscribe en un género textual determinado en el caso tratado, el ensayo se vincula necesariamente a una práctica social y comúnmente utilizada en el ámbito escolar y académico. Esta práctica discursiva se caracteriza por el tipo de discurso, en esta oportunidad la argumentación como modo de organización del discurso en el género ensayo. Visto de esta forma, la práctica discursiva se inserta en modos y tipos, con relación a ello, Serrano (2005a), propone que:

Una práctica discursiva (La cual moviliza modos y tipos discursivos y géneros textuales), se encuentran en tres planos interrelacionados: el de la enunciación (el cual se inscribe el enunciador y el enunciatario), el del enunciado (mediante el 
cual el enunciador se dirige al enunciatario) y el del referente (al cual refiere el enunciador mediante el enunciado). Enunciador, enunciatario y referente son roles discursivos inscritos en el texto (pág. 91).

En efecto, esta formulación plantea que la construcción discursiva llevada a cabo por estos componentes y que se origina en la generación de un texto que un oyente o un lector interpreta, esto permite una interrelación entre el enunciador, el enunciatario y referente que dan cuenta de qué es lo que se propone argumentar en un texto bajo las propiedades de coherencia y cohesión, a través de la interpretación del referente.

Debe señalarse que para el autor antes mencionado el enunciador y referente asumen roles lingüísticos, cognitivos y evaluativos que se complementan recíprocamente a través del intercambio de información en la que la interpretación juega un papel realmente importante.

Por otra parte, la argumentación y la narración son dos modos discursivos que se ponen al servicio uno del otro en las prácticas comunicativas ya sean orales o escritas, en la temática planteada que es el ensayo la narración del referente ocasiona que se entrelacen argumentos basados en valores axiológicos, estéticos, afectivos y razonables. En palabras de Martínez (2005c): "El reconocimiento del enunciado como espacio común donde se construyen y evidencian las fuerzas enunciativas, ofrece una explicación de la construcción tridimensional del sujeto discursivo: axiológico (Ethos), emotivo (Pathos), razonable (Ratio)" (pág. 11).

Sin duda, la argumentación en el género ensayo colabora en la construcción discursiva de la identidad del sujeto. Parafraseando a Serrano, (2005b): "producir y acrecentar la adhesión suponen dos procesos discursivos diferentes: el primero, cuando se obtiene la adhesión de alguien a una tesis, es ocasionar en ese alguien una trasformación identitaria, trasforma sus creencias, sus valores" (pág. 98). 
En el segundo caso, el proceso discursivo acrecienta la adhesión de alguien a una tesis si se garantiza en el discurso argumentado sus creencias, valores y afectos. Bajtin (1979), citado en Martínez (2005d): "el enunciado se plantea como la única unidad que puede posibilitar la construcción de la metáfora del mundo, es decir, la presentación del mundo interpretado en el discurso" (pág. 13); esta relación intersubjetiva hace que se ponga de manifiesto la identidad del sujeto su visión del mundo y la compresión de los fenómenos del entorno.

\subsection{El Género Ensayo}

Se considera para este trabajo como una producción escrita con unas particularidades propias de un trabajo académico y se presenta como el resultado de una revisión documental previa sobre una temática cultural, histórica, literaria científica, social, entre otros. Para Díaz (2004a): "el ensayo es un trabajo intelectual por tratar sobre un tema, un problema, una propuesta o un fenómeno con originalidad, libertad, coherencia y elegancia literaria. Su origen puede ubicarse en el renacimiento con los trabajos de Montaigne y Voltaire" (pág. 108). Parafraseando a Díaz (2004b): "un ensayo puede ser un trabajo corto que debe reflejar dominio del tema, competencia lógica y competencia comunicativa, su construcción puede combinar la descripción, narración, exposición y argumentación" (pág. 110).

Al respecto de la elaboración del género ensayo en el contexto educativo es frecuente y el propósito del mismo en el aprendizaje puede incidir favorablemente en la promoción del trabajo intelectual independiente y en la libertad de opinión, propiciar el desarrollo del pensamiento crítico reflexivo, divergente, convergente y creativo, estimula la producción de conocimientos significativos, posibilita el desarrollo ético y estético del estudiante al reconocer los aportes de otros, ayuda a organizar los conocimientos. 


\subsection{La argumentación y el ensayo}

La argumentación y el ensayo representan una actividad académica de mucho valor en las prácticas educativas, a través de ellos se logra producir textos con originalidad y pertinencia, en el que el estudiante demuestra su habilidad para exponer sus ideas sobre algún tema con argumentos válidos a un posible lector o destinatario.

Para esta investigación se define el género ensayo como una labor académica que estimula el aprendizaje independiente, la libertad de opinión promociona la reflexión, la crítica, la autonomía en la escritura y la creatividad. Vale señalar que en la escritura y reescritura de ensayos se utiliza la argumentación como modo de organización discursiva.

En atención a lo anterior para la elaboración de ensayos se debe considerar la coherencia, el orden, la precisión, la originalidad, el aparato crítico, la revisión de textos, la interpretación, el análisis, la argumentación con ejemplos y aportes, además ofrecer conclusiones sobre la temática trabajada en el escrito.

\subsection{Proceso de la enseñanza de la escritura}

En la actualidad los enfoques pedagógicos en la enseñanza de la escritura sugieren que no se debería considerar el proceso de la escritura como una técnica estándar donde el individuo descifre reproduciendo y repitiendo lo observado, al contrario, la escritura como una actividad cognitiva en la que el individuo construye el texto con una finalidad clara y definida.

Para tal efecto, el acto de escribir cumple con varias etapas desde el momento que la persona tiene intención de expresar sus ideas o pensamientos y plasmarlas en un papel. Según Cassany (2008a): "los procesos de composición son todo lo que se piensa, hace y se escribe, el autor desde que tiene la intención de escribir un texto hasta la versión final” (pág. 175). Así, el autor antes señalado, describe las estrategias cognitivas utilizadas por el 
escritor al momento de escribir un texto hasta la versión final, que en este proceso de construcción propone ideas, busca referentes, organiza, redacta, y en esta elaboración se fomenta el estilo del escritor.

En función de lo anterior, Jolibert (2008): establece fases para el proceso de escribir, que implica la relación docente estudiante, estas se describen a continuación:

1). Iniciar la producción de un texto a partir de un contexto oral y con significado para el niño; 2). Establecer los elementos relevantes en la composición escrita (a quién le escribo, con qué propósito lo hago, qué es lo que quiero decir); 3). Realizar en la primera escritura individual un esbozo completo del tema; $4)$. Confrontar las primeras escrituras entre los alumnos del grado; 5). Confrontar la escritura con expertos como es el caso del maestro facilitando en las formas del discurso o en la gramática textual (conectores, subtítulos, tiempos verbales, entre otros); 6). Reescritura individuales, parciales o globales; 7). Revisar la versión final del texto; 8). Evaluar el texto producido. (pág. 166).

Se puede aseverar que la facilitación del docente en el proceso de la adquisición de la escritura es una labor de importancia en la formación del individuo desde sus primeros inicios escolares y que se lleva a lo largo de la vida, además se establece en el individuo la capacidad para expresar sus ideas, pensamientos y conocimientos del mundo a través del registro escrito.

En tal sentido, Quintero, Molina y Di Gravia (2017), aportan lo siguiente: "la escritura ocupa un sitial preponderante en los procesos de formación académica y por este motivo los responsables de orientar ese aprendizaje deben involucrarse, muy comprometidamente en todo lo que implique mejorar las estrategias de enseñanza" (pág. 33); cómo se puede evidenciar, la práctica académica se fortalece con la aprehensión del conocimiento a través de las habilidades y destrezas para leer y escribir, por tal motivo es necesario que los enseñantes de la lengua ejecuten estrategias significativas para el aprendizaje 
de la misma.

\subsection{Construcción del género ensayo y la escritura}

Escribir corresponde a una de las habilidades del lenguaje pareada con leer, al respecto, Odreman (2005), explica: "escribir es un hecho lingüístico cuya esencia consiste en producir significados a través de un sistema de signos gráficos estables" (pág. 24); no obstante, la escritura representa un creativo y complejo proceso que da respuesta a la necesidad de la humanidad para trascender, de contar vivencias para la posteridad y de hacer conocer las ideas, los pensamientos y sentimientos a través de un código y de la comunicación o del lenguaje.

Por el contrario, a lo expuesto, la escuela como han aportado Bettelheim y Zelam (1983), citados en Gutiérrez (2010): "despoja a la escritura... de esa cualidad creadora, transformadora y liberadora que es inherente a la palabra escrita" (pág. 25); así en el nivel de media general en el área de lengua la escritura de ensayos con argumentos sólidos en el discurso escrito no se genera.

En este sentido, la escritura de acuerdo con Flower y Hayes (1996), citados en Marín (2006a), es un proceso estructurado en etapas: preescritura, escritura y revisión consideradas "momentos recursivos que se imbrican, se superponen, se repiten y vuelven sobre si mismos una y otra vez. Esos momentos, además, interactúan con los conocimientos y las habilidades lingüísticas de los que escriben" (pág. 277); en relación con la cita, conviene señalar que el sujeto que escribe pasa por los tres procesos.

Siguiendo ese orden de ideas, la preescritura corresponde a la fluidez de pensamientos, conocimientos que se hallan en la mente del escritor, que se amplían con la lectura de diferentes materiales tales como: la conversación

entre pares, la vista de un video o película e incluso escuchar música o salir de paseo. En función de ello, Serrano, Peña, Aguirre, Figueroa, Madrid, y 
Cadenas (2002a): consideran que la preescritura, la planificación o preparación de un escrito "implica una serie de operaciones...se recopila un material, se lee, se selecciona y se descarta aquello que no guarda relación con lo que queremos desarrollar" (pág. 25).

En este componente de la escritura; Calkins (1997), citado en Serrano, et al. (2002b), describen la fase de preparación de la escritura como aquella en la que "los escritores trazan posibles líneas de desarrollo para sus textos, o diseñan un recorrido para sus ideas" (pág. 25). Esta tarea de planificación de puede realizar de forma individual pero no excluye realizar en equipos que permita el intercambio de opiniones para nutrir el texto. Por otra parte, Cassany (2008b), explica lo siguiente:

En el proceso de planificación los escritores se forman una representación mental de las informaciones que contendrá el texto. Esta representación es muy abstracta. No es necesariamente un esquema completo y desarrollado. En algunos casos, una palabra clave puede representar una cadena completa de ideas, además, no es preciso que sea verbal: puede ser una imagen visual (pág. 175).

Dicho de este modo, es el escritor quien decide qué idea, expresión o tema abordará el texto en este subproceso de escritura. Seguidamente, se revela la textualización que la define Flower y Hayes (1996), citados en Marín (2006b): "esencialmente en el proceso de convertir las ideas en lenguaje visible" (pág. 285); describiendo la naturaleza peculiar de la tarea de escribir porque al elaborar el primer borrador, aun cuando es el pensamiento del autor el que se plasma o se traduce, seguramente no se hace con las convenciones requeridas. Según Smith (1982), citado en Serrano, et al. (2002c): el escritor se permite "cambiar de lugar una palabra, añadir otra, tachar una idea, ampliarla y/o desarrollarla de otra manera" (pág. 26).

Con base a lo formulado, en la construcción de la argumentación como 
modo de organización del discurso del género ensayo en el contexto de estudio permitirá a los estudiantes hacer uso de los procesos de la escritura para planificar sus ideas, plasmarla, reescribirlas para darle mejor sentido a las ideas que el escritor pretende adherir al auditorio, al enunciatario 0 enunciatarios. De este modo, el escritor confirma que el texto expresa realmente con claridad lo que él desea comunicar en el discurso, como lo expone Marín (2006c): "el que escribe va produciendo cambios, cuya profundidad y cantidad depende... de su grado de pericia" (pág. 289).

Dentro de este contexto importa que la revisión textual permita al escritor dar forma al texto, ajustarlo a los propósitos previstos, adaptarlo al auditorio, entre otras. En función de ello, los estudiantes deberían comprender el proceso de la escritura de ensayos bajo la argumentación la importancia de la revisión del texto tantas veces como sea posible.

Ahora bien, conviene expresar que los procesos de escritura no se dan de forma lineal o como etapas sucesivas sino más bien, recursivas, lo que permite al escritor ir y venir en los pasos ya descritos. De tal manera, que compete al docente llevar a cabo acciones y propuestas idóneas para que el estudiante se acerque en la práctica de la escritura a la producción de ensayos con argumentos elaborados con coherencia y cohesión bajo las técnicas de la argumentación como modo de organización del discurso.

\section{Conclusiones}

Los planteamientos expuestos reflejan la importancia pedagógica que tiene la argumentación con relación al alcance de competencias y habilidades en el desarrollo integral de los jóvenes para que se expresen de forma coherente y con propiedad sobre los aprendizajes o conocimientos escolares que conforman la academia.

Sin embargo, si no se utiliza la argumentación como modo de 
organización discursiva incidirá en la apropiación y empoderamiento del lenguaje como una construcción social que permita a los estudiantes y profesores crear la posibilidad de interactuar en múltiples situaciones y experiencias que se proyectan en la expresión oral o escrita del estudiante y en su proceso de formación. Martínez (2005e), señala:

El género discursivo está relacionado con los grados de complejidad de las sociedades, pues sintetiza la relación entre práctica social y uso del lenguaje en una situación específica y esta, por tanto, relacionado con la evolución misma de las sociedades y el lenguaje (pág. 59).

Según lo expresado por la autora, la utilización del discurso argumentado es de gran utilidad y relevancia para los sujetos intersubjetivos que aun sin percatarse de ello, habitualmente están elaborando, organizando y estructurando discursos, más aún en la labor académica en la cual constantemente se plasma o registra los diversos enunciados y producciones textuales.

Esto representa que la argumentación y el ensayo están indisolublemente ligados a las prácticas educativas como un elemento comunicativo de gran importancia. Ahora bien, esto ocasiona una relación mutua entre los interlocutores, que partiendo de la argumentación como modo de organización discursiva y de la didáctica de la lengua se hace consiente en el entorno educativo. Es así como los sujetos discursivos asumen una posición crítica y reflexiva frente a un discurso oral o escrito.

\section{Referencias}

Andarcia, R. (2006). Origen y tendencias del estudio del Ethos: de Aristóteles el análisis del discurso. Textura (CETEX), 5(8), 29-41. ISSN: 1317-5920.

Cassany, J. (2008a,b). La cocina de la escritura. Barcelona, España: Anagrama. 
Calsamiglia, H., \& Tusón, A (2001). Las cosas del decir. España: Editorial Ariel.

Charaudeau, P. (2004a,b). La Problemática de los géneros. De la situación a la construcción social. Revista Signos, 37(56), 23-39, ISSN: 07180934. Recuperado de:

http://dx.doi.org/10.4067/S0718-09342004005600003

Díaz, D (2004a,b) Como se elabora un ensayo. Acción pedagógica, 13(1), 108-113. Recuperado de:

http://www.saber.ula.ve/bitstream/123456789/17148/2/documento2.pdf

Gutiérrez, M. (2010). Mundos posibles para leer y escribir en la escuela:

Biografía y autobiografía. Colección Brújula Pedagógica, 2. Caracas, Venezuela: Editora El Nacional.

Jolibert, J. (2008). Interrogar y producir textos auténticos vivencias de aula. II parte. Chile: Dolmen-Estudio.

Marín, M. (2006a,b,c). Lingüística y enseñanza de la lengua. La enseñanza de la lengua. Larousse. Biblioteca Didáctica, 5. México: Larousse.

Martínez, M. (2005a,b,c,d,e). La construcción del proceso argumentativo. Cátedra UNESCO para la lectura y la escritura. Cali, Colombia: Universidad del Valle.

Odreman, N. (2005). Estrategias para el desarrollo de las competencias comunicativas. Colección Brújula Pedagógica, 02. Caracas, Venezuela: Editora El Nacional.

Perelman, Ch. (1997a,b). El imperio retórico. Colombia: Editorial Norma.

Quintero, G., Molina, T., \& Di Gravia, A. (2017). Competencias de Escritura en Estudiantes de la Carrera de Educación. (Caso: Universidad Pedagógica Experimental Libertador. Mérida, Venezuela). Revista Scientific, 2(3), 31-52. Recuperado de:

https://doi.org/10.29394/scientific.issn.2542-2987.2017.2.3.2.31-52

Serrano, E. (2005a,b). Didáctica del Discurso: Narración, Argumentación 
y construcción de identidad. Cátedra UNESCO para la lectura y la escritura. Colombia: Universidad del valle.

Serrano, S., Peña, J., Aguirre, R, Figueroa, P., Madrid, A., \& Cadenas, I. (2002a,b,c). Formación de lectores y escritores autónomos. Orientaciones didácticas. Mérida, Venezuela: Universidad de Los Andes y Fe y Alegría, Zona los Andes. 


\section{Magdaly Carolina Méndez Omaña}

e-mail: mgdy.m41@gmail.com

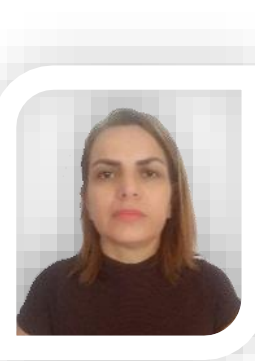

Nacida en Mérida, Venezuela. Magister Scientiarum en Gerencia Educativa de la Universidad Nororiental Privada "Gran Mariscal de Ayacucho" en agosto del año 2016. Especialista en Evaluación Educacional en la Universidad Valle del Momboy en marzo del año 2009. Licenciada en Educación de la ULA en diciembre del año 2006. Licenciada en Letras de la ULA en Mención Lengua y Literatura Hispanoamericana y venezolana en septiembre del año 2001. Docente activa del Ministerio del Poder Popular para la Educación, facilitadora del Seminario de Investigación de la UVM, asesor, tutor y jurado de trabajos especiales de grado.

El contenido de este manuscrito se difunde bajo una Licencia de Creative Commons ReconocimientoNoComercial-Compartirlgual 4.0 Internacional 